\title{
El trabajo colaborativo en red impulsor del desarrollo profesional del profesorado
}

\author{
MARIA JOSÉ NAVARRO MONTAÑO
} Universidad de Sevilla, Sevilla, España

ANTONIA LÓPEZ MARTÍNEZ Universidad de Sevilla, Sevilla, España

MARIA ELENA HERNÁNDEZ DE LA TORRE Universidad de Sevilla, Sevilla, España

\section{RESUMEN}

En este artículo se presenta un estudio de caso sobre una estrategia de aprendizaje colaborativo entre profesionales de la educación en un ámbito específico de trabajo, el alumnado con autismo, para apoyar el intercambio de experiencias a través de las redes. La muestra se compone de un equipo de 11 profesoras de aulas de autismo específicas, cuyo trabajo se basa en diseñar y contrastar estrategias educativas de actuación para este alumnado. La recogida de datos se ha realizado a través de entrevistas en grupo, cuestionarios y análisis del Plan de Trabajo. Los resultados indican que el trabajo planificado y consensuado, con una trayectoria común de participación continuada en el tiempo, ofrece una garantía de calidad que camina hacia la mejora en educación. Así, el grupo de trabajo ofrece seguridad profesional a sus participantes, favorece la consecución de objetivos comunes y evita el aislamiento profesional de los profesores en aulas específicas, ya que la comunicación en la red es la base de su trabajo.

\section{PALABRAS CLAVE}

aprendizaje colaborativo; redes educativas; redes profesionales; comunicación en red; educación de calidad. 


\title{
COLLABORATIVE WORK IN RED DRIVE PROFESSIONAL DEVELOPMENT OF TEACHERS
}

\begin{abstract}
This paper exposes some findings obtained by a research on collaborative learning. Its general purpose was to gain a deeper knowledge on the effect of networks in teacher's collaborative learning. The sample was conformed by 11 teachers in autism specific classrooms, whose work consists in designing and contrasting educational intervention strategies for those students. Data collection was performed through group interviews, questionnaires and analysis of the work plan. The results indicate that the work planned and agreed with a common path of continued participation, provides a basis to the improvement of education. Given that the working group provides professional security for its participants, helps to achieve common objectives and avoid professional isolation of teachers in specific classrooms, network communication is the basis of their work.
\end{abstract}

KEYWORDS

collaborative learning; educational networks; professional networks; network communication; quality education.

\section{TRABALHO COLABORATIVO EM REDE NO DESENVOLVIMENTO PROFISSIONAL DE PROFESSORES}

RESUMO

Este artigo apresenta um estudo de caso sobre uma estratégia de aprendizagem colaborativa entre profissionais da educação em uma área de trabalho específica, o autismo, para apoiar o intercâmbio de experiências através das redes. A amostra é composta por uma equipe de 11 professoras de aulas específicas para autistas, cujo trabalho é baseado na concepção e contraste de estratégias de intervenção educativa para estes alunos. A coleta de dados foi realizada através de entrevistas em grupo, questionários e análise do Plano de Trabalho. Os resultados indicam que o trabalho planejado e consensual, com um caminho comum de participação continuada, fornece uma garantia de qualidade em direção à melhoria da educação. Assim, o grupo de trabalho oferece segurança profissional aos seus participantes, favorece a realização de objetivos comuns e evita o isolamento profissional de professores em aulas específicas, uma vez que a comunicação em rede é a base do seu trabalho.

\section{PALAVRASCHAVE}

aprendizagem colaborativa; redes de ensino; redes profissionais; comunicação em rede; educação de qualidade. 


\section{INTRODUCCIÓN}

En este artículo presentamos un modelo de trabajo compartido y colaborativo entre profesores que trabajan en una red escolar, y que, partiendo de sus propias necesidades, promueven un proyecto de trabajo coordinado que propicia la mejora y el cambio en los centros escolares que participan para la atención a la diversidad del alumnado a través del intercambio de ideas, reflexiones, prácticas y procesos de trabajo en los centros. Hemos analizado el funcionamiento de algunas redes sociales (Carbonai y Colvero, 2014) para tenerlas como referente en el funcionamiento de los centros educativos, donde la participación de los profesores en las "redes educativas" supone un cambio en relación a la reflexión que realizan acerca de sus prácticas, lo que representa, no solo una forma diferente de trabajar, sino que implica cambios culturales entre profesionales y organizaciones.

La formación entre compañeros de trabajo lleva al aprendizaje colaborativo entre profesionales y se entiende como un proceso de aprendizaje en "feedback", ya que cada participante aporta conocimiento al grupo y a la red de la que forma parte, se forma en el grupo y vuelve de nuevo al grupo de trabajo para su puesta en común a través de prácticas consensuadas. En este contexto de aprendizaje se potencia que los participantes exterioricen y compartan algunos conocimientos desarrollados a través de su práctica profesional, como señala Basque (2013). Básicamente, en las redes se autogestiona un trabajo compartido en base a una comunicación desarrollada en un espacio de trabajo común, lo que genera a su vez un conocimiento compartido. El uso de las TIC ha tenido una función muy importante en el desarrollo de estas redes, constituyéndose en un medio de comunicación que propicia el intercambio de información y la elaboración conjunta de los conocimientos. En este sentido, es necesario incidir en la capacidad de aprendizaje a través de la red. Como señala Vilar (2008, p. 271), "la capacidad de aprendizaje en la red es un sistema que se autorregula en función de las experiencias que va teniendo, incorpora información y construye nuevos significados compartidos.". La filosofía de trabajo en la red se basa en pasar del trabajo individual e independiente, de un "trabajo solitario", a "trabajar de una forma integrada con los demás.” (Vilar, 2008, p. 273).

Aprender en red supone establecer un entramado de relaciones e interrelaciones de significados conceptuales y prácticos entre los componentes de la red, de esta forma, estas redes trabajan continuamente en el ámbito educativo para crear conocimiento. Un ejemplo de este entramado de relaciones lo encontramos en el aula entre los alumnos, los cuales crean su red de relaciones denominadas "redes naturales". De la misma forma, cuando los profesores proponen estrategias de aprendizaje en grupo, cada uno aporta sus conocimientos estableciendo una forma de aprender entre iguales. Domènech (2010, p. 52) a partir de sus estudios sobre el trabajo en redes, ha establecido una serie de elementos que las caracterizan:

- las redes son irregulares en su entramado, son diversas, flexibles, cambian y se mueven; se hacen y se rehacen continuamente por las incorporaciones y las bajas y los aportes que realiza. 
- las redes son de constitución horizontal, lo que propicia el respeto, la confianza y el clima de respeto e intercambio.

- las redes son flexibles y abiertas en su aprendizaje para que funcione como red, sintiéndose como miembros de una comunidad.

La teoría sobre redes y el aprendizaje colaborativo en la actualidad ha pasado de una idea centrada en la creación del conocimiento de forma individual a la construcción del conocimiento a partir y desde las redes profesionales. Todo ello ha supuesto en la escuela la reivindicación de una forma de trabajar en educación de una manera diferente, esto es, trabajar juntos, de manera complementaria y cooperativa, para trabajar mejor. En este sentido nos preguntamos cómo puede ayudar el trabajo en red a la mejora de la escuela. Si partimos de la base de la red como un espacio de aprendizaje compartido, avanzamos hacia la cultura de la colaboración y a compartir conocimiento en la red, incorporando nuevas experiencias al trabajo por la mejora de la educación. Trabajar en red se considera hoy en día una ventaja y un beneficio para la mejora, ya que el intercambio de información, como señala Rué et al. (2005, p. 406), “enriquece a todos los miembros del grupo de trabajo y da lugar a nuevos puntos de reflexión y mejora en la práctica diaria". Los participantes de las redes escolares aportan nuevas perspectivas que, en la mayoría de las ocasiones, son extrapolables a las realidades de cada centro. Asimismo afirma Rué et al. (2005, p. 407), "el hecho de reunirse en un grupo numeroso y con distintos puntos de vista, hace más valiosas las discusiones sobre los temas tratados". Otros autores como Dias (2012), abordan la importancia de la formación del profesorado en relación con la atención a la diversidad cultural y las implicaciones de estos procesos de trabajo claborativo para la educación infantil.

El trabajo en red, por tanto, tiene como objetivo prioritario crear una coordinación estable de centros con el propósito de instituir, asimismo, una red de comunicación y trasvase de experiencias de unos centros a otros. Se trata de construir una red de intercambio, de relación y de cooperación, entendiendo estas redes como aquellas que promueven la generación de procesos innovadores y participativos de las personas que comparten el proceso educativo. Es imprescindible, por tanto, conocer la trayectoria de cada red y sus procesos de reflexión para pasar posteriormente al marco de cada centro en concreto, estudiando cómo estan influyendo en el desarrollo y aprendizaje de los propios centros. Por otra parte, las redes educativas escolares se basan en un concepto de formación sostenible para toda la comunidad educativa (Rué et al., 2005) en condiciones de igualdad y equidad, estableciendo relaciones de carácter horizontal entre todos los profesores. Es una formación para todos vinculada a la igualdad de oportunidades, la mejora y la formación. El objetivo del trabajo en red, en definitiva, trata de mejorar nuestra práctica educativa y combatir la lucha contra la exclusión del alumnado, la mejora y la formación de la práctica educativa para el profesorado y para la comunidad escolar.

En otros trabajos recientes hemos estudiado el funcionamiento y desarrollo de las redes educativas de centros escolares, incidiendo en el impacto y la repercusión en la comunidad educativa (Hernández et al.,2012a-b). También hemos analizado el trabajo colaborativo que realizan las redes de centros (López et al., 2013) y su alcance en la mejora educativa. 


\section{JUSTIFICACIÓN Y OBJETIVOS DE LA INVESTIGACIÓN}

El estudio que presentamos se inscribe en un proyecto en curso denominado "Estudio de las Redes Educativas de Centros Escolares (ReCes) para la promoción y desarrollo de una educación para la equidad y la participación" en el que colaboramos como investigadoras de la Universidad de Sevilla con Centros de Profesores (CEPs) de Sevilla y provincia. Los objetivos de este estudio de caso se enmarcan en el análisis del trabajo en red y son los que a continuación se relacionan:

- Estudiar el funcionamiento de la red: organización, distribución de tareas, temporalización, Plan de Trabajo, etc.

- Profundizar sobre las repercusiones que tiene la participación del trabajo en la red en cada centro.

- Estudiar el impacto que tiene la participación de cada centro en esta red.

- Establecer nuevas líneas de investigación a partir de los resultados obtenidos en el estudio (conclusiones).

El estudio del caso único conlleva algunas características que compartimos en este análisis: es interpretativo, los significados son interpretados partiendo de la observación y la voz de los protagonistas; es naturalista, capta las realidades y las acciones en la forma en que estas se presentan y suceden; la selección de la muestra es deliberada, no aleatoria ni probabilística; el diseño es flexible y emergente, adaptándose a las condiciones cambiantes en las que se produce el proceso de investigación; en el proceso investigador, es observador de los escenarios naturales y establece contacto directo con los protagonistas; el marco de referencia desde el que se observa es siempre personal y es el que da sentido a la situación; es particular, no generaliza, se dirige a situaciones particulares singulares (Bolívar, Domingo y Fernández, 2001).

En el desarrollo de nuestro estudio hemos seguido el procedimiento secuencial descrito por Creswell (2009) en los siguientes pasos:

- Paso 1: correo e-mail para petición de cita al Centro de Profesores.

- Paso 2: entrevistas semiestructuradas a los asesores de los centros de profesores en bloques de preguntas y grabadas en audio.

- Paso 3: cuestionarios para la recogida de datos a los asesores y equipos directivos.

- Paso 4: análisis de los datos.

- Paso 5: codificación de los datos.

- Paso 6: agrupamiento de los datos.

- Paso 7: interpretación de los datos.

Para la recogida de datos del trabajo de esta red hemos utilizado entrevistas en grupo (focus-group), cuestionarios y análisis de documentos/Plan de Trabajo. Este análisis se ha realizado desde una óptica descriptiva, ya que se detallan las reflexiones de los participantes en la red. Esta metodología está centrada en un enfoque de investigación-acción, donde la recogida y análisis de datos se encuentra en línea con posiciones de pensamiento actual como es la filosofía comunitaria, en la que se inscribe el proyecto. 
Los instrumentos utilizados para la recogida de datos son los presentados en la Tabla 1.

Esta muestra está constituida por una red de centros ya consolidada, con seis años de experiencia, y en la que participan once centros de Educación Infantil y Primaria de la provincia de Sevilla. Se trata por tanto de un estudio de caso único que trabaja para la inclusión de alumnos autistas en los centros escolares.

\section{RESULTADOS}

En la Tabla 2 presentamos los resultados proporcionados por los instrumentos de recogida de datos para el análisis del trabajo de esta red.

Respecto a las entrevistas en grupo a los asesores sobre el "trabajo en red" Hemos analizado las afirmaciones obtenidas de modo literal bajo el esquema de trabajo presentado en la Tabla 2.

Respecto al Cuestionario para Asesores - Los resultados del análisis de datos del cuestionario para asesores sobre esta red son los siguientes.

El comienzo y constitución de esta red tiene como punto de partida la necesidad de dar respuesta al trabajo con el alumnado autista en centros ordinarios. El objetivo ha sido y es trabajar de forma coordinada en distintos centros algunos aspectos básicos de los procesos de enseñanza-aprendizaje de este alumnado: la competencia lingüística, expresión escrita y posteriormente la lectura. Participan 11 centros de Primaria y Secundaria con aulas específicas, profesorado de PT y tutores, así como algunos profesores y directores de estos centros. La trayectoria de trabajo de esta red es de seis años, por lo que, en general, la acogida que tiene en los centros esta red es positiva, con una participación muy dinámica y expectativas muy altas para el aprendizaje de este alumnado y su inclusión escolar y social.

Esta red pretende afrontar la convivencia escolar desde una perspectiva social y posteriormente educativa, por ello plantea, inicialmente, necesidades de formación de sus componentes. El objetivo es ofrecer atención especializada a este alumnado, favoreciendo una respuesta común y coordinada de todo el centro, no solo de los especialistas. El contenido de este proceso responde al siguiente esquema de trabajo:

- Adecuación de la atención educativa e inclusión de este alumnado en los centros.

Tabla 1 - Instrumentos de recogida de datos.

Instrumentos de recogida de datos

Entrevistas en grupo (Focus-Group) de carácter dialógico sobre el tema de discusión "El trabajo en red". Participantes: dirección y asesores de Centro de Profesores, investigadoras de la red de centros

Cuestionario semi-estructurado e individual para asesores de redes: "Cuestionario para asesores de Centros de Profesores sobre Redes Educativas de Centros Escolares"

Cuestionario estructurado: "Cuestionario para Equipos Directivos" de los centros participantes en la red

Análisis de documentos: Plan de Trabajo 
- Realización de propuestas por centros sobre cómo trabajar las técnicas instrumentales.

- Reflexión sobre la práctica partiendo de documentos de apoyo.

- Introducción de mejoras en el aula llegando a acuerdos en el centro.

Tabla 2 - Asesores Centro de Profesores. Primeras evidencias del debate (aproximación al concepto real)

\begin{abstract}
"el trabajo en red implica un proceso no improvisado, fraccionado en fases guiadas ..."
"las redes escolares cuentan con recursos desarrollados y analizados, y gestionan el tiempo ..." /

"las redes seleccionan contenidos..." / "las redes aportan algunos indicadores de seguimiento de evaluación, una metodología de trabajo" / "es una forma de compartir para asumir así buenas prácticas de aula" / " en las redes de trabajo es muy importante el proceso de reflexión final" / "encuentras redes de trabajo que se convierten en red de apoyo profesional, casi personal o de acompañamiento..." / "sirven para compartir entre profesionales sus mismas penalidades, sus mismas inquietudes ..." “es retroalimentación constante..."
\end{abstract}

\title{
Trabajo con redes (experiencias)
}

\begin{abstract}
"los centros educativos que participan en trabajos en redes son aquellos que les preceden prácticas innovadoras ..." / "participan colectivos donde existe un cierto clima de trabajo colaborativo, que tengan una inquietud de trabajo en equipo y trabajo en grupos ..." / "las personas que trabajan con redes escolares son personas que quieren superar la parte del aislamiento" / "no existe una correlación directa entre el profesorado que le gusta el trabajo en equipo y que le guste el trabajo en red, hay muchos profesionales que trabajan bien en red, porque les encantan el tema de las redes sociales ..." / "incorporando actividades en la redes relacionadas con la atención a la diversidad, se generaliza que todo el profesorado vea que es algo básico y transversal ..." / "a lo mejor no hay un centro que presente una buena práctica completa, pero sin embargo con las pequeñas muestras de varios centros sí se puede hacer ..." / "cuando se trabaja con familia y centro educativo de manera simultánea y positiva, la vida del centro se va normalizando, porque los padres se implican en la participación ..."
\end{abstract}

\section{Consenso argumental (conclusiones, ideas nuevas)}

"en cada centro hay personas que se anotan a la red arrastrando al centro ..." / "siempre se piensa que primero existe una reunión física y posteriormente aparecen las redes, aunque es al contrario" / "la red permite que las políticas educativas pongan valor a algunas necesidades del sistema que por sí mismas no surgirán ..." / "la participación en red propicia que los proyectos educativos sean más inclusivos" / "suele llamar la atención los silentes de las comunidades o dinamizador silente, que son organizadoras del proyecto pero no quieren participar en ellos de forma visible, es decir, evitan el protagonismo ..." / "el papel de participación de las familias está aún por determinar ..."

\section{Conclusiones}

Es muy importante que se den los motivos para que el trabajo cooperativo y en red se produzca. Una de las cuestiones de la red es acabar con el aislamiento del docente en su propia aula, y la muerte metodológica y de atención a todos los alumnos.

Si no hay una participación de las familias en la cual la presencia en el centro sea visible, la coherencia entre lo que decimos y lo que hacemos no está garantizada.

Es importante implicar a las familias de forma positiva durante el proceso de aprendizaje del estudiante, con la finalidad de participar de manera inclusiva en cualquier aspecto del centro.

Las redes de trabajo generan gran cohesión grupal, incrementando la confianza y el fortalecimiento de relaciones satisfactorias.

Las redes escolares generan oportunidades de aprendizaje a los profesionales.

Nota: Síntesis de información registrada a través de la entrevista en grupo en el Centro de Profesores a los asesores de redes escolares. 
En general, el grupo de profesionales que impulsa este trabajo, apuesta $y$ cree en el trabajo en equipo del profesorado, con lo que ha nacido como una necesidad frente al aislamiento que, en ocasiones, suele tener este profesorado en los centros. Sin embargo, los asesores señalan su enorme dificultad de coordinación y de toma de decisiones en aspectos metodológicos. Los Equipos Directivos, respecto a la participación en estas redes de Centros, se posicionan de forma muy positiva, demandando formación para que las iniciativas se expandan a nivel del centro y en toda la comunidad educativa, con una implicación total en el trabajo y tareas que se desarrollan. Aunque algunos están muy motivados, reconocen que otros lo hacen porque la inspección está implicada. La red dispone de apoyos ofrecidos por la Plataforma Moodle y de documentos proporcionados por el CEP en lo que se refiere a recursos materiales. En recursos humanos dispone del equipo asesor del CEP, inspector y coordinadores de los Equipos de Orientación Educativa.

En lo que se refiere a la distribución de tareas, reuniones, organización interna en red, los asesores responden que esto se realiza a través de grupos de trabajo en los centros que funcionan a lo largo del curso y mediante la elaboración de planes de convivencia. Se convocan reuniones con los representantes de los ciclos, los jefes de estudio, los inspectores y los asesores de referencia. En ellas siempre hay un orden del día en el que se especifica el tema a tratar.

La coordinación de la red en general recae en los asesores implicados de los CEPs y la coordinadora del grupo de trabajo, ya que el grupo se compone exclusivamente de mujeres. En cada centro se implican los Equipos de Ciclo, los responsables de convivencia, coordinadores del "proyecto escuela", coordinadores del grupo de trabajo, etc. La comunicación entre los miembros de la red se desarrolla a través del foro de la red instalada en Moodle, en reuniones y mediante el uso de los recursos informáticos, reuniones presenciales de grupos de trabajo y correos electrónicos.

Los procesos de participación en red se desarrollan mediante un trabajo en común, tales como el diseño de fichas tipo, guiones para focalizar el trabajo de los diferentes ciclos en reuniones y la puesta en común de metodologías a seguir. Los conflictos se abordan en las reuniones, dedicando mucho tiempo al debate, y mediante el seguimiento y evaluación, introduciendo medidas de mejora que el proceso aconseje. La formación que demandan los centros que participan en la red son cursos presenciales, el grupo de trabajo como modalidad auto-formativa, además de cursos, jornadas, congresos etc.

En lo que se refiere a la participación en cada centro, los asesores señalan que existe una mayor relación entre los Equipos Directivos de los centros a través de muchas reuniones de coordinación, ya que "el proceso mueve y dinamiza aspectos de la vida del centro". Se han iniciado "tímidos pasos" para relacionarse con otras localidades a partir de la puesta en común de algún trabajo compartido mediante reuniones conjuntas inter-localidad, para compartir iniciativas que se están llevando a cabo con profesorado de otros centros y otros profesionales o instituciones que trabajan la temática. Respecto a las familias, estas se implican como colaboradores externos en actividades solicitadas o a través de actividades 
o eventos puntuales que el centro realiza. Los representantes municipales en general no participan, en algún caso un representante de la inspección y algunos trabajadores sociales.

Respecto al impacto de la participación en la red, los asesores creen que han dinamizado y contribuido a la toma de decisiones coordinadas, así, el profesorado y su implicación educativa con el alumnado ha cambiado totalmente. Es muy positivo, ya que se tiene otra mirada de la convivencia, ha mejorado la participación, se han dinamizado procesos curriculares y se ha implicado a familias, etc. El profesorado acoge a este alumnado con autismo de otra manera y presenta evidencias de algunas propuestas de cambio en la práctica del aula. Esta participación en red ha mejorado la formación del profesorado, transformado actitudes, resuelto conflictos, sistematizado procesos, detectado necesidades y mejorado la participación y trabajo en equipo.

Esta red no tiene un Plan de Seguimiento, encontrándose, sin embargo, en Fase de Consolidación. Se encuentran actualmente planificando una sesión de seguimiento e iniciando contacto con otras redes.

Entre los obstáculos destacan los normales en este tipo de procesos: falta de tiempo a veces, la vinculación-compromiso con algunos profesionales del centro y familias, escasez de recursos económicos y la participación en formación, sesiones de trabajo y seguimiento. Destacan la implicación y dinamismo del grupo de profesionales comprometidas lo que ha dado validez y fundamentación a los planteamientos de esta red, siendo la toma de decisiones conjunta fruto de la reflexión colectiva. La satisfacción ha sido compartir inclusión y mejora del alumnado en los centros y el trabajo en grupo de estas profesoras.

Respecto al Cuestionario para Equipos Directivos de los centros pertenecientes a esta red - Hemos organizado los resultados en torno a las categorías de análisis presentadas a continuación.

En relación a la participación, el 100\% de equipos directivos de estos centros conocen la existencia de este grupo de trabajo, el 60\% afirma que revierte en la práctica educativa y entienden que la formación del grupo es esencial para su desarrollo, para el reciclaje del profesorado y el desarrollo de la calidad educativa (4CA). El trabajo inter-centros es muy interesante en un grupo consolidado y estable, con una dinámica de trabajo en línea con las necesidades del aula: "me parece bien puesto que repercute en la intervención con el alumnado" (10CA).

Respecto a los cambios/mejoras, los equipos directivos afirman que "los conocimientos adquiridos y los materiales elaborados se utilizan en la intervención con el alumnado" (10CA). Aunque los cambios a nivel de aula difieren en cada centro, siempre se ponen de manifiesto en las prácticas y en la mejora de las relaciones entre el profesorado (1CA). Se ha adoptado una metodología de trabajo basada en proyectos (2CA) y "se ha mejorado la coordinación entre el profesorado de las diferentes aulas, unificando criterios a seguir... se enriquece la cantidad y calidad de actividades propuestas y el ambiente de trabajo es más saludable" (3CA). Otro cambio se relaciona con la organización y el funcionamiento del aula de autismo, está más estructurada y se han incluido rutinas que mejoran el rendimiento del alumnado (4CA, 7CA). La coordinación con las aulas de autismo de otras zonas educativas se 
considera muy positiva (5CA), "se percibe una mayor seguridad a nivel de trabajo con este alumnado" (8CA).

En lo que se refiere al profesorado, los equipos directivos manifiestan que el "profesorado de los centros se encuentra muy implicado desde el principio" (2CA) en un $80 \%$ de los centros, proponiendo "actividades de sensibilización para fomentar el conocimiento del autismo" (3CA). El profesorado se encuentra más concienciado, aunque es necesaria una formación básica ( $5 \mathrm{CA}$ ), ya que es una labor que implica a todo el profesorado y a toda la institución. Es necesario trabajar en equipo de forma coordinada y consensuada en el propio centro, así, el profesorado "se siente con menos 'miedos' a la hora de integrar en su aula de estos alumnos" (8CA). Los profesores más interesados son aquellos que están relacionados con este alumnado, esto es, "profesoras de PT, profesoras de AL, educadoras, monitoras, tutores/as." (9CA).

La participación del centro en la red se ha regulado a través del Plan de Formación del Profesorado y "dentro del Plan de Formación del profesorado, incluido en el Proyecto Educativo, se da prioridad a aquella formación directamente relacionada con estos alumnos... se publican los cursos y las jornadas y se entrega en mano a los profesionales más cercanos a la problemática planteada” (5CA), para ello "se pone a disposición el aula y sus recursos para los diferentes componentes del grupo, y flexibiliza el horario de exclusiva si alguna reunión lo requiere” (3CA). En un 20\% el profesorado es consciente de la falta de tiempo para poder participar, pudiendo ocurrir que "solamente participe la tutora del aula específica y es en un día en el que no hay exclusiva” (1CA).

En cuanto a las mejoras, en el 100\% de los casos estas se han producido en la atención al alumnado trabajando en actividades comunes en diferentes aulas que integran a estos alumnos (1CA), optimizando los espacios de inclusión en aulas ordinarias y demás espacios educativos (recreos, sala de psicomotricidad, actividades de creatividad, etc.). El objetivo es la inclusión como prioridad (6CA) y el "conocimiento más acertado de estos alumnos" (8CA) a la hora de relacionarse con ellos tanto los docentes como el alumnado (8CA). Se trata de "crear condiciones óptimas de convivencia, los alumnos incluyen en sus rutinas la integración con los demás estableciendo pautas comunicativas relacionadas con las habilidades sociales." (5CA). Así, las respuestas del equipo directivo apuntan dos puntos de vista diferentes: en un porcentaje muy pequeño (10\%) afirman que las mejoras consideran "no creen que sean necesarias, pues su funcionamiento ya está regulado y supervisado por el propio CEP" (9CA) y, por otro lado, y en un porcentaje muy amplio (90\%) están abiertos a cualquier cambio que redunde en la mejora del centro "siempre que sea necesario o requieran nuestra colaboración estaremos disponibles" (4CA). Estas mejoras "incluyen en todas las actividades del centro a estos alumnos, se implican en las rutinas comunicativas, intentando dar solución a todas las demandas... mantenemos canales abiertos y fluidos con otras instituciones, (psicólogo escolar, autismo Sevilla.") (5CA).

Análisis de Documentos: Plan de Trabajo - El Plan de Trabajo de esta Red de Autismo se encuentra estructurado de la siguiente forma (Tabla 3). 


\section{DISCUSIÓN DE RESULTADOS}

A continuación presentamos la discusión de los resultados obtenidos organizados en torno a los siguientes temas de interés para la investigación:

- El trabajo colaborativo en red revierte en la mejora educativa: la puesta en funcionamiento y el desarrollo de esta red de centros escolares ha surgido a través de la creación de un grupo de trabajo colaborativo constituido por profesorado de aulas de autismo. Los asesores se han implicado activamente en la puesta en marcha de este grupo de trabajo con la intención de poner en funcionamiento un proyecto concreto que responda a las necesidades educativas de este alumnado. Desde sus inicios la red tiene la finalidad de mejorar la calidad educativa de los centros participantes, aunque inicialmente solo se trataba de abordar mejoras parciales que afectaban a un reducido número de estudiantes y en el que se implicaban algún profesorado. Inicialmente se creó con tímidas aportaciones para la mejora de los centros y en ámbitos concretos como la metodología y las estrategias didácticas. Los profesionales implicados en la puesta en funcionamiento de la red son el profesorado, los Equipos Directivos, asesores, orientadores e Inspección Educativa. La acogida que tiene en la mayoría de centros la red es positiva, con una participación muy dinámica, teniendo los centros expectativas muy altas con los proyectos puestos en marcha debido a la trayectoria de varios años de trabajo de sus participantes.

- La red responde a las necesidades de los centros, mediante el trabajo colaborativo: el profesorado que Directivo considera muy positiva esta participación, incluso demandan formación para que la iniciativa se extienda a nivel del Centro y en toda la comunidad educativa. La implicación del equipo directivo en el trabajo y tareas que se desarrollan es total, así lideran el proceso, dinamizan, hacen partícipe al resto del centro

Tabla 3 - Plan de trabajo del grupo de autismo.

\begin{tabular}{l}
\hline \multicolumn{1}{c}{ Análisis de documentos: Plan de trabajo } \\
\hline 1. Título y breve descripción. "El aprendizaje basado en Proyectos con alumnado con TEA" \\
\hline 2. Situación de partida que justifica la constitución del Grupo de Trabajo: antecedentes, justificación, \\
grado de incardinación con el Proyecto de Centro, proyección en la práctica docente \\
\hline 3. Plan del Proceso de Trabajo: fase previa (competencias docentes, prácticas de aula), fase de desarrollo \\
(ámbito de autoformación, ámbito de transferencia en el aula), evaluación (indicadores de mejora de \\
competencias docentes, indicadores de innovación de las prácticas de aula) \\
\hline 4. Calendario de reuniones y horario de las mismas \\
\hline 5. Producción de documentos y materiales educativos: ámbito de la formación, ámbito de la \\
transferencia en el aula, de evaluación utilizada \\
\hline 6. Recursos y apoyos \\
\hline 7. Integrantes del grupo de trabajo \\
\hline
\end{tabular}


y establecen canales de comunicación, trabajan de forma colaborativa, comparten experiencias y reflexiones, estableciendo estrategias de trabajo en equipo, como son reuniones de ciclo y/o reuniones inter-centros. $\mathrm{El}$ equipo apoya a los asesores junto con los docentes. Es esencial conocer las necesidades de los centros y las necesidades planteadas por los asesores, ya que tienen relación directa con la temática de interés que se están desarrollando en el equipo de trabajo: atención especializada al alumnado con autismo y unidad de inclusión, favoreciendo una respuesta común y coordinada de todo el centro, no solo de los especialistas; la programación, metodologías y evaluación en Competencias Básicas que faciliten su incorporación al currículo.

- Concreción de temas para desarrollar el trabajo colaborativo en red: la actividad de la red se centra en el estudio de temas concretos para mejorar la escolarización de este alumnado. Algunos de estos temas son los siguientes: atención educativa e inclusión del alumnado con autismo en los centros educativos, programación y evaluación en competencias que revierta en la mejora de la inclusión de estos alumnos, coordinación de líneas pedagógicas de todos los centros y establecimiento de aspectos metodológicos, organizativos y curriculares comunes para cada centro y mejora de la práctica docente, evaluación formativa, evaluación de calidad y mejora de los centros, análisis de resultados trimestrales, pautas para la elaboración de la programación didáctica en las diferentes áreas curriculares, mejora del clima de centro y de aula, y reflexión sobre la práctica partiendo de una serie de documentos de apoyo, introducción de mejoras en el aula y acuerdos de centro.

- Desarrollo de reuniones periódicas presenciales en los centros para trabajar en colaboración: en cuanto a la distribución de tareas, reuniones, organización interna en red, etc., estas se inician con una sesión presencial en la que se forma el grupo de trabajo que funcionan a lo largo del curso y se nombra un coordinador que informa al resto del profesorado. Se elabora un calendario consensuado con el equipo asesor y el servicio de inspección para llevar a cabo una estructura de las actuaciones sistematizadas en reuniones puntuales con representantes de ciclos, jefes de estudio y asesores. Se establece un orden del día especificando el tema a desarrollar. La coordinación de la red recae en los asesores implicados de los CEPs y coordinador/a del grupo de trabajo.

- La tecnología educativa, recurso para promover el trabajo colaborativo y establecer una comunicación virtual permanente: es necesario establecer una diferencia entre la comunicación presencial y no presencial. La primera se desarrolla en reuniones del grupo de trabajo a través del diálogo y el contacto personal; la comunicación no presencial se desarrolla en todos los centros a través del foro de la red instalada en el Moodle y mediante el uso de los recursos informáticos como el correo electrónico y el foro de debate instalado en la plataforma, lo que le ofrece al profesorado implicado un contacto continuo. El uso de las tecnologías 
educativas se entiende como un recurso valioso ya que permite mantener un contacto permanente con los representantes de los centros en la red. Gairín y Castro (2010) también describen un modelo de trabajo en red que permite crear, compartir y gestionar conocimientos, mediante el uso intensivo de la red, denominada, Red Atenea, entre diferentes personas, principalmente, directivos de centros de Educación Primaria y Secundaria, tanto directores y directoras, como jefes y jefas de estudio, en definitiva estudiosos de temas de liderazgo educativo.

\section{CONCLUSIONES}

Los resultados nos permiten evidenciar que el trabajo colaborativo entre profesionales genera conocimientos compartidos, promueve la adquisición de experiencias y apoya el desarrollo profesional de los profesores más allá del aula. Se trata de un aprendizaje intencional como proceso de intercambio de conocimientos y proceso de creación de los mismos. Asimismo, este análisis de los resultados obtenidos nos permite, por una parte, realizar una evaluación y valoración de los centros que están trabajando en esta red de autismo y, por otra, conocer el trabajo colaborativo de la propia red. Hemos organizado las conclusiones en torno a los siguientes temas de interés para la investigación:

- La red educativa de centros escolares ha impulsado la colaboración entre profesionales: la colaboración entre profesionales es un principio básico para contribuir a la mejora en educación, ya que los cambios culturales que la escuela está viviendo en la actualidad necesita del trabajo colaborativo entre profesionales. En este contexto, la participación a través del trabajo en red, se concibe como prioridad y es una nueva forma de trabajo para los profesionales, necesitando asimismo superar las dificultades que ha supuesto el trabajo individual de los profesores en los centros escolares. El trabajo en red es una apuesta sólida desde la que orientar la educación del futuro e impulsar ámbitos y proyectos de trabajo compartidos con otros sectores, instituciones y entidades de la comunidad educativa. El trabajo en red aumenta la capacidad de actuación de los profesionales implicados y promueve el trabajo colaborativo a nivel de grupos. Podemos concluir que el hecho de formar parte de la red anima a la participación a gran parte del profesorado aunque a veces no logra atraer a la totalidad del Claustro, si bien los primeros que se interesan son los profesionales que trabajan con alumnado con autismo.

- La participación en la red tiene una incidencia positiva en los centros implicados a distintos niveles: la participación de los centros en la red tiene una incidencia positiva en el funcionamiento de cada uno de ellos, sobre todo en temas relacionados con la educación de este alumnado, así como con la educación en valores, desarrollo del currículum, atención a la diversidad, formación del profesorado, trabajo en equipo, coordinación a distintos niveles, procesos de reflexión sobre la propia práctica, convivencia en el centro, procesos de enseñanza-aprendizaje, 
desarrollo de competencias concretas, metodología y selección de materiales curriculares, resolución de conflictos, detección de necesidades y sistematización de procesos de trabajo. Todo ello tiene una influencia directa en el funcionamiento general de los centros y además repercute en la intervención del profesorado en el aula, elaborando información útil y recursos adecuados.

El trabajo en red trata de iniciar en los centros el denominado ciclo de transformaciones para el diseño, desarrollo e implicación de todos en las innovaciones y mejoras. El desarrollo de estos planes de mejora por una parte y de proyectos concretos por otra, incide en la organización y el funcionamiento de los centros y tiene una repercusión directa en las prácticas de aula que acogen al alumnado con autismo.

- El trabajo colaborativo en red promueve el desarrollo de proyectos de centro más inclusivos: el desarrollo de proyectos de centro inclusivos surge ante la necesidad de diseñar proyectos educativos orientados a mejorar la convivencia y la atención a todos en los centros y se concibe como texto sustentador de la tarea pedagógica (Argos, Ezquerra y Castro 2010). El profesorado implicado es el que trabaja con alumnado con autismo y, como consecuencia, la participación en la red incide directamente en todos aquellos aspectos vinculados a la atención a la diversidad. Los proyectos se diseñan principalmente para este alumnado y se desarrollan a nivel de centro. La idea de inclusión supone hacer a las escuelas responsables de todos sus estudiantes, por lo que los Proyectos de Centro inclusivos suponen un cambio de las estructuras, relaciones y prácticas educativas. Estos proyectos que abordan el diseño de procesos de inclusión utilizan estrategias didácticas para la diversidad, estableciendo apoyos para las necesidades educativas de este alumnado.

- El trabajo en colaboración a través de la red aumenta el sentido de pertenencia a la comunidad: el trabajo en red se concibe como un espacio abierto a la participación de profesionales, organizaciones y grupos de innovación orientados a la mejora de la educación. La red desarrolla mejoras en cada centro, atiende a la diversidad y es un factor clave para el cambio escolar. Esto es apoyado por investigaciones recientes sobre Proyectos en Red, como la de Muñoz (2005), que pone de manifiesto que las redes se convierten en organizaciones que aumentan la capacidad de actuación, mejoran la eficacia de los procesos, se supera la toma de decisiones de tipo vertical, ya que la participación es horizontal, aumentan el sentido de pertenencia a la comunidad y se toma conciencia de la capacidad para participar y modificar una realidad concreta y mejorarla.

En definitiva, los centros que trabajan en colaboración aumentan su capacidad de mejora, son capaces de llevar a cabo una evaluación de necesidades, se agrupan para participar y cooperar en innovaciones; la participación se establece en cada departamento y el consenso se produce con más facilidad. En esta línea de trabajo, Stornaiuolo, Dizio y Hellmich (2013, p. 81-82), refiriéndose al desarrollo 
comunitario, han realizado un estudio en el que vinculan las redes sociales con los jóvenes y las escuelas en el que afirman que "la comunidad es el proceso activo y fluido de comunicación, así, las nuevas formas mundiales de comunicación que permiten las tecnologías móviles y digitales ofrecen posibilidades para "nuevas formas de pertenencia".

- El trabajo en colaboración a través de la red contribuye a disminuir el aislamiento profesional: el trabajo en colaboración a través de la red les permite a algunos profesionales, que apuestan por la innovación y el cambio, salir del aislamiento endémico de algunas escuelas, mientras se ponen en cuestión las estructuras rígidas y jerarquizadas del sistema educativo, y se activan nuevas formas de liderazgo y nuevos modos de aprendizaje en colaboración. El profesorado es el protagonista principal de su propio proceso de formación, en este sentido son destacables, por una parte, los procesos de autoevaluación basados en las prácticas docentes, y por otra, los procesos de autoformación en los que está inmerso el profesorado participante en la red.

La formación del profesorado se inscribe en procesos de formación continua y en procesos de formación específica, en función de las necesidades concretas que presenta cada centro y la comunidad. El profesorado encuentra en la red actitudes favorables a los procesos de formación, garantizándola a través de las relaciones continuas que se establecen dentro de las mismas. La participación en redes desarrolla en el profesorado un proceso formativo en autonomía y responsabilidad y en este sentido los autores Ruiz et al. (2012) señalan que la formación en autonomía y responsabilidad es uno de los objetivos de la educación del siglo XXI. Por consiguiente, en los centros que participan en redes se produce un aumento del trabajo en colaboración, de estudio y reflexión sobre temas educativos generales y específicos.

Las mejoras de la participación en red se concretan en las siguientes: las redes se convierten en organizaciones que aprenden y aumentan la capacidad de actuación; mejoran la eficacia de los procesos, superando obstáculos para la mejora; la participación es horizontal, salvando la toma de decisiones de tipo vertical; aumentan el sentido de pertenencia a la comunidad, superándose la no pertenencia al grupo de trabajo y el aislamiento; se toma conciencia de la capacidad para participar y modificar una realidad concreta y mejorarla, superando las barreras para la inclusión y la equidad.

\section{PROPUESTAS DE MEJORA}

Teniendo presente el estudio llevado a cabo, realizamos a continuación algunas propuestas de mejora, sobre las que seguir trabajando, con el objetivo de avanzar en el estudio de las redes:

- Reconocida la autonomía de los centros para participar en redes, se deberían llevar a cabo autoevaluaciones periódicas orientadas a promover mayor participación de los centros y atraer al profesorado que no participa. 
- Estudiar en profundidad los intereses que comparte el profesorado implicado en la red, vinculados a las necesidades de formación en ámbitos concretos.

- Analizar en profundidad las mejoras llevadas a cabo en los centros para su difusión.

- Profundizar en el estudio de la gestión de la red, como aspecto importante a considerar para garantizar su continuidad.

- Realizar un estudio comparativo con otras redes, ya consolidadas también, con objeto de recabar propuestas que incidan en la mejora de su funcionamiento.

\section{REFERENCIAS}

Argos, Javier; Ezquerra, Pilar, Castro, Ana. El proyecto educativo como elemento vertebrador de la práctica pedagógica: reflexiones y propuestas para la acción. Teoría de la Educación, Salamanca, v. 22, n. 2, p. 183-205, 2010.

BAsQue, Josianne. Apoyar el desarrollo profesional continuo del personal académico a través del intercambio de experiencias. Revista de Universidad y Sociedad del Conocimiento, v. 10, n. 1, p. 116-134,2013. Consultado el 18 de Octubre en http://dx.doi.org/10.7238/ rusc.v10i1.1572

Bolívar, Antonio; Domingo, Jesús y Fernández, Manuel. La investigación biográfico-narrativa en educación. Madrid: La Muralla, 2001.

Carbonai, Davide; Colvero, Ronaldo, B. Papéis sociais no ensino médio. Uma análise basada na teoría das redes. Revista Brasileira de Educação, v. 19, n. 58, p. 671-689, jul-set. 2014.

CRESWELL, John. Research design: qualitative, quantitative and mixed methods approaches (3rd edition). Thousand Oaks: Sage Publishing, Inc., 2009.

DiAs, Lucimar, R. Formação de professors, educação infantil e diversidades étnico-racial: saberes e fazeresnesse proceso. Revista Brasileira de Educação, v. 17, n. 51, p. 661-749, set-dez., 2012.

Domènech, Josep. Aprendiendo en red. Aula de Innovación Educativa, Editorial Graó, v. 142, p. 51-54, 2010.

Gairín, Joaquín; Castro, Diego. Situación actual de la dirección y gestión de los centros de enseñanza obligatoria en España. Revista Española de Pedagogía, Madrid, v. 247, p. 401-416, 2010.

Hernández Elena; López, Antonia; Navarro, Ma José, y Ordoñez, Rosario. Funcionamiento y desarrollo de redes educativas de centros escolares: impacto y repercusiones en la comunidad educativa. Congreso Innovación y Organización de Instituciones Educativas CIOIE, Universidad de Granada, España, 2012a.

. Study of school's educational networks: keys aspects for their analysis and improvement from the assessment of teacher's advisors centers. Congreso ECER, Universidad de Cádiz, España, 2012b. 
López, Antonia; Ordoñez, Rosario; Hernández, Elena; Navarro, Ma José. Funcionamiento de redes educativas de centros escolares. Desarrollo de un trabajo colaborativo. Revista Española de Orientación y Psicopedagogía, v. 24, n. 1, p. 25-41, 2013. Muñoz, José L. Redes educativas locales: un nuevo reto en la formación. IV Congreso de Formación para el Trabajo, Zaragoza, 9-11 Noviembre, 2005.

Navarro, Ma José; Hernández, Elena; Ordoñez, Rosario; López, Antonia. Las redes educativas de centros escolares: plataformas para la mejora educativa. Congreso Internacional INFAD, Abril, Moscú-San Petesburgo, 2013.

Rué, Joan; Balaguer, Lourdes; Forastiello, A. M.; García, Alicia; Moreno, Francesc X.; NúñEz, Carmen et al. El desarrollo de la profesionalidad docente mediante redes. Revista Iberoamericana sobre Calidad, Eficacia y Cambio en Educación, v. 3, n. 1, p. 403-411, 2005.

Ruiz, Marta; Bernal, Antonio; Gil, Fernando; Escámez, Juan. Ser uno mismo: repensando la autonomía y la responsabilidad como coordenadas de la educación actual. Teoría de la Educación, Salamanca, v.24, n. 2, p. 59-81, 2012.

Stornaiuolo, Amy; Dizio, Jennifer K.; Hellmich, Emily A. Desarrollando la comunidad: jóvenes, redes sociales y escuelas. Comunicar, Revista Científica Iberoamericana de Comunicación y Educación, Andalucía (España), v. xx, n. 40,p.78-88, 2013. Consultado el 20 de Noviembre en http://dx.doi.org./10.3916/C40-2013-02-08 VILAR, Jesús. Implicaciones éticas del trabajo en red y la acción comunitaria. Cultura y Educación, Barcelona, v. 20, n. 3, p. 267-277, 2008.

\section{SOBRE LAS AUTORAS}

Maria José Navarro Montaño es doctora en Filosofía y Ciencias de la Educación por la Universidad de Extremadura (España). Profesora de la Universidad de Sevilla (España).

E-mail: maripe@us.es

Antonia López Martínez es doctora en Filosofía y Ciencias de la Educación por la Universidad de Sevilla (España). Profesora de la misma institución. E-mail: anlomar@us.es

Maria Elena Hernández De La Torre es doctora en Filosofía y Ciencias de la Educación por la Universidad de Sevilla (España). Profesora de la misma institución.

E-mail: eht@us.es

Recibido en 8 de enero de 2015

Aprobado en 14 de agosto de 2015 